\title{
MODELO DE AVALIAÇÃO DO EMPREGO DAS FERRAMENTAS DO LEAN PRODUCT DEVELOPMENT NO PROCESSO DE DESENVOLVIMENTO DE PRODUTOS
}

Gustavo Scheid Prass (guto.prass@gmail.com) - Centro Tecnológico de Joinville, Universidade Federal de Santa Catarina.

Cristiano Vasconcellos Ferreira (cristiano.v.ferreira@ufsc.br) - Centro Tecnológico de Joinville, Universidade Federal de Santa Catarina.

\section{RESUMO}

As empresas que procuram garantir sua participação em um mercado gradativamente mais competitivo, têm o desafio de desenvolver seus produtos de maneira dinâmica e eficiente. Neste contexto, a inserção de ferramentas do Lean Product Development (LPD) no processo de desenvolvimento de produtos (PDP) de uma organização é uma atividade complexa, que requer mudanças comportamentais e culturais. Deste modo, a avaliação do emprego destas ferramentas contribui com informações para implantar melhorias no PDP. Este trabalho tem como principal objetivo, a construção de um modelo de avaliação que possibilita classificar o emprego das ferramentas do LPD no processo de desenvolvimento de produtos. $O$ modelo proposto avalia o emprego de 10 ferramentas do LPD, com o auxílio de 40 critérios de avaliação. Cada critério recebeu um peso com auxílio do método de tomada de decisão multicritério MACBETH, o que garantiu que os critérios fossem ponderados por ordem de importância. O modelo de avaliação proposto pode ser aplicado em diversos setores da indústria, sendo que a forma com que este foi construído permite a inserção de outras ferramentas do LPD, se houver necessidade. E por fim, os resultados obtidos proporcionam às empresas participantes uma visão global do desempenho no emprego das ferramentas do LPD no PDP, o que promove informações pertinentes para a melhoria de seus processos de desenvolvimento.

Palavras-chave: Modelo de Avaliação; Ferramentas e Métodos; Lean Product Development

Área: Lean Product Development

\section{INTRODUÇÃO}

Inúmeras organizações têm se esforçado para acelerar o seu processo de desenvolvimento de produtos (PDP), tal que a concorrência é global, em inúmeros casos. A empresa que produz e entrega as demandas ao cliente, com o nível de qualidade exigido, no prazo correto e com o custo adequado, tem o espaço para potencializar o seu negócio. E, para isso, ter um PDP estruturado é fundamental (DAL FORNO; FORCELLINI, 2012; MACHADO, 2006).

O PDP é um conjunto de atividades que buscam encontrar especificações de projeto de um produto a partir das necessidades de mercado e da capacidade tecnológica, indo ao encontro com as estratégias competitivas da empresa. Sendo, assim, uma interface entre a empresa e o mercado, identificando oportunidades e propondo soluções (ROZENFELD et al., 2006). O PDP é dividido em fases, apresentadas de forma seqüencial na Figura 1, mas que podem ocorrer de forma simultânea em inúmeras aplicações. 
Figura 1. Visão Simplificada do Modelo de Referência do Processo de Desenvolvimento de Produtos. Fonte: Adaptado de Rozenfeld et al. (2006).

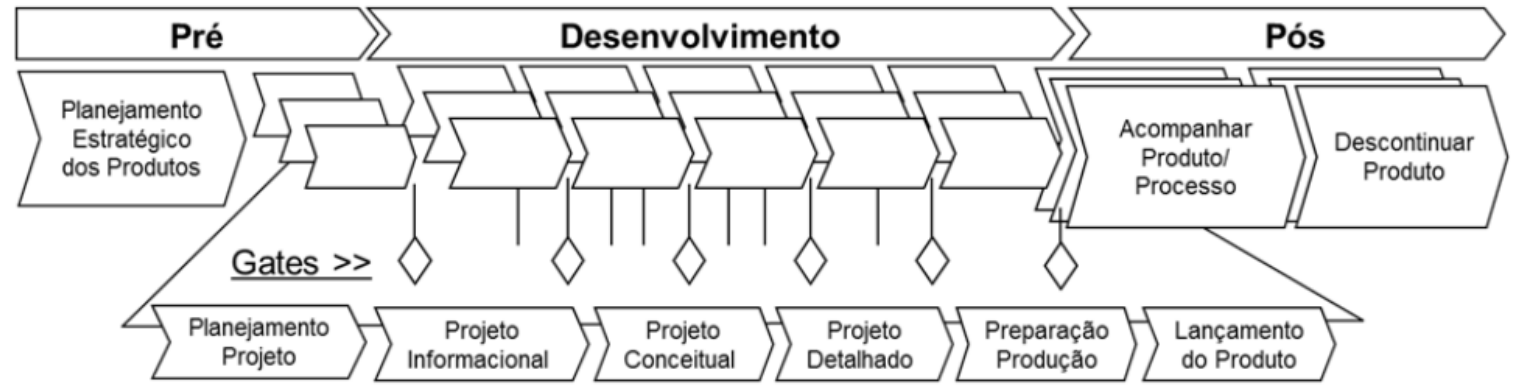

Para aumentarem a sua competitividade, algumas práticas têm sido adotadas pelas organizações. Entre as práticas, destaca-se o Lean Manufacturing, que aplica os princípios enxutos na manufatura. Por sua vez, os princípios enxutos também são aplicados no PDP, o que é chamado de Lean Product Development (Desenvolvimento Enxuto de Produtos) (LEÓN; CROSS, 2011; OEHMEN; REBENTISCH, 2010).

Para Nakamura (2010), o LPD por si só não representa uma sistemática estruturada para o PDP, desta forma não existe um modelo de referência construído sob os princípios do LPD. Desta forma, este trabalho propõe um modelo de avaliação do emprego de ferramentas do Lean Product Development no Processo de Desenvolvimento de Produtos, que busca mensurar o desempenho do emprego dessas ferramentas pela indústria. Assim, pretende-se contribuir com a indústria, uma vez que as ferramentas do LPD possibilitam a minimização de desperdícios, aumentam as chances de sucesso de um projeto, minimizam o tempo e o custo de desenvolvimento, resultando num aumento de competitividade.

Na Figura 2 são descritas as fases desta pesquisa científica em forma de um diagrama, apresentando as principais atividades de cada fase. A primeira fase é a revisão da literatura, onde são explorados os princípios e as ferramentas do LPD. A segunda fase é a proposição do modelo de avaliação, onde são definidos os critérios de avaliação do emprego das ferramentas do LPD no PDP, atribuídos os pesos para cada critério, definidas as faixas de classificação e criado o formulário de avaliação. A terceira fase é a aplicação do modelo de avaliação, onde o formulário de avaliação é aplicado na indústria, levantando informações sobre o emprego das ferramentas do LPD. Finalizando com a quarta fase, conclusões, onde são apresentados os resultados, as contribuições e recomendações para trabalhos futuros.

Figura 2. Metodologia Científica Empregada. Fonte: Adaptado de Prass (2017).

Fase 1

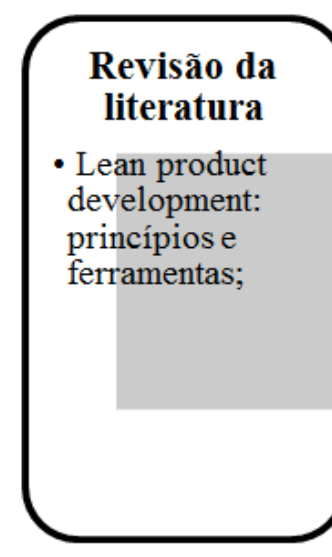

Fase 2

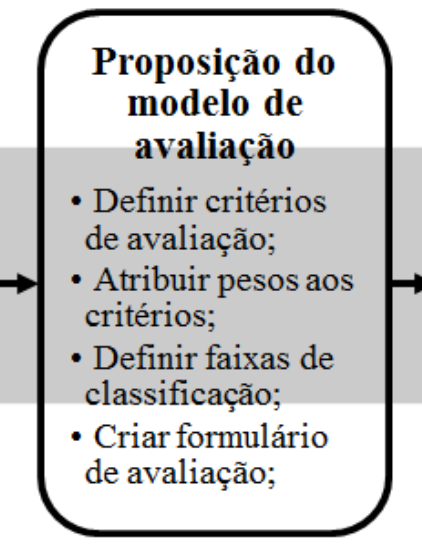

Fase 3

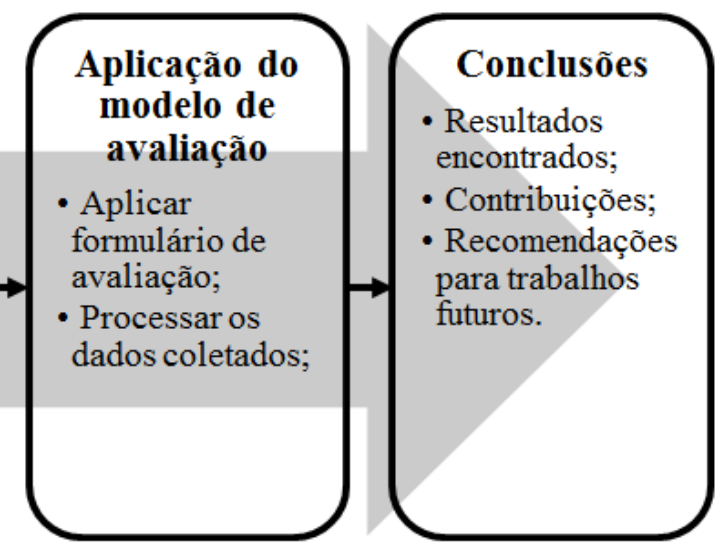




\section{LEAN PRODUCT DEVELOPMENT}

Uma das abordagens citadas na literatura de desenvolvimento de produtos é o Lean Product Development, que tem como característica buscar a criação de valor e a eliminação de desperdícios, alinhando os princípios enxutos com as ferramentas de desenvolvimento de produtos (MILAN; REIS; COSTA, 2015; DAL FORNO; FORCELLINI, 2012).

O LDP pode ser definido como: métodos e ferramentas de desenvolvimento multifuncionais que são governadas pelos princípios enxutos e podem ser usadas para maximizar o valor e eliminar desperdícios no PDP (LEÓN; CROSS, 2011). O LPD busca eliminar tudo aquilo que não é benéfico ao sistema, de forma a eliminar e/ou evitar desperdícios. $O$ foco da abordagem é no cliente e nos processos que agregam valor em termos de preço, qualidade, prazos e entrega, incluindo aspectos sociais e ambientais (DAL FORNO; FORCELLINI, 2012).

A base para o LPD são os princípios enxutos, que buscam aproveitar completamente os investimentos feitos em pessoas, equipamentos, infraestrutura e materiais para criar valor aos clientes; podendo postergar/conter investimentos a curto prazo e aumentando a produtividade, tornando-se uma empresa mais competitiva. Entende-se o LPD como uma maneira de produzir mais com menos (DAL FORNO; FORCELLINI, 2012).

\subsection{Princípios Enxutos}

Os princípios enxutos são empregados com sucesso em diversas áreas de conhecimento, como Lean Manufacturing, Lean Enterprise e Lean Product Development. De modo geral, existem cinco princípios enxutos: especificar o valor, identificar o fluxo de valor, desenvolver o fluxo, trabalhar o sistema puxado e buscar a perfeição (RAUCH; DALLASEGA; MATT, 2016; MILAN; REIS; COSTA, 2015; DAL FORNO; FORCELLINI, 2012). Porém, segundo León e Cross (2011), não existe um consenso sobre os princípios enxutos, podendo haver diferenças entre autores.

Os princípios enxutos precisam ser traduzidos em ferramentas para serem aplicados de maneira direta nos processos de uma empresa. De acordo com Milan, Reis e Costa (2015), aplicar os princípios enxutos no PDP precisa ser visto como uma estratégia econômica e o LPD deve ser tratado como indispensável para aumentar a competitividade e a lucratividade.

O emprego dos princípios enxutos no PDP é um processo difícil, devido à natureza dinâmica, a grande interação com demais atividades da empresa e pela quantidade de informações geradas no decorrer deste processo. Todavia, incluir os princípios enxutos no PDP, na forma de ferramentas do LPD, o torna mais ágil, eficaz e flexível para receber novas demandas de mercado (SALGADO; MELLO; SILVA, 2009).

\subsection{Ferramentas do LPD}

As ferramentas do LPD são implementadas para melhorar os resultados de uma organização, satisfazendo as necessidades dos clientes e das demais partes interessadas, traduzindo os princípios enxutos em algo aplicável (DAL FORNO; FORCELLINI, 2012). León e Cross (2011) afirmam que as ferramentas do LPD compreendem, um ou mais, princípios enxutos e que não existe uma lista fixa, nem para os princípios e nem para as ferramentas do LPD.

Na Tabela 1 são descritas algumas ferramentas do LPD, selecionadas nos trabalhos de Dal Forno e Forcellini (2012) e León e Cross (2011). 
Tabela 1. Ferramentas do LPD.

\begin{tabular}{|c|c|c|}
\hline Ferramenta & Descrição & Fonte* \\
\hline $\begin{array}{l}\text { Engenharia } \\
\text { Simultânea }\end{array}$ & $\begin{array}{l}\text { Abordagem sistemática para o desenvolvimento integrado e paralelo do projeto } \\
\text { de um produto e os processos relacionados, incluindo manufatura e suporte. É } \\
\text { um processo de gerenciamento que examina múltiplos aspectos de um produto, } \\
\text { com uma equipe multidisciplinar. }\end{array}$ & $\begin{array}{l}{[1],[2],} \\
{[3] \quad \mathrm{e}} \\
{[4]}\end{array}$ \\
\hline $\begin{array}{l}\text { Engenharia } \\
\text { Simultânea } \\
\text { Baseada em } \\
\text { Conjuntos }\end{array}$ & $\begin{array}{l}\text { Cada membro da equipe de desenvolvimento do produto desenvolve um } \\
\text { conjunto de alternativas paralelas e independentes. As alternativas são avaliadas } \\
\text { entre si e eliminadas, até que a melhor opção permaneça, a qual é gerada pela } \\
\text { combinação de sistemas e componentes do produto. }\end{array}$ & $\begin{array}{l}{[1],[5],} \\
{[6] \quad \mathrm{e}} \\
{[7]}\end{array}$ \\
\hline $\begin{array}{l}\text { Mapeamento do } \\
\text { Fluxo de Valor }\end{array}$ & $\begin{array}{l}\text { Tem como objetivo representar o estado atual de um processo para visualizar } \\
\text { alguns desperdícios e calcular tempos. As melhorias são planejadas a posteriori, } \\
\text { no mapa de estado futuro e no plano de ações. }\end{array}$ & $\begin{array}{l}{[8],[9]} \\
\mathrm{e}[10]\end{array}$ \\
\hline $\begin{array}{l}\text { Gerenciamento } \\
\text { Visual }\end{array}$ & $\begin{array}{l}\text { Oferece um feedback imediato, cuja finalidade é fornecer informações } \\
\text { acessíveis e de forma simples capazes de facilitar o trabalho diário, reforçando a } \\
\text { autonomia dos funcionários. }\end{array}$ & $\begin{array}{l}{[1] \quad \mathrm{e}} \\
{[11] .}\end{array}$ \\
\hline Repo & $\begin{array}{l}\text { Ferramenta que busca identificar a situação atual, a natureza do problema, as } \\
\text { contra medidas possíveis, a melhor contra medida, como colocá-la em prática e } \\
\text { a evidência de que o problema foi efetivamente solucionado, escrito em apenas } \\
\text { uma das faces de uma única folha de papel A3. }\end{array}$ & $\begin{array}{ll}{[1]} & \mathrm{e} \\
{[12] .} & \end{array}$ \\
\hline $\begin{array}{l}\text { Biblioteca de } \\
\text { Projeto }\end{array}$ & $\begin{array}{l}\text { Plataforma que tem como propósito ser a memória da empresa onde o registro } \\
\text { das lições aprendidas deve ser documentado e utilizado como meio facilitador } \\
\text { para novos projetos além de se tornar um guia para orientar as atividades } \\
\text { envolvidas em toda a cadeia do processo. }\end{array}$ & $\begin{array}{ll}{[1]} & \mathrm{e} \\
{[13] .} & \end{array}$ \\
\hline Padronização & $\begin{array}{l}\text { ocessos, de modo que estes } \\
\text { rão. }\end{array}$ & {$[1]$} \\
\hline $\begin{array}{l}\text { Simulação } \\
\text { Virtual }\end{array}$ & $\begin{array}{l}\text { Utilização de softwares como auxílio para a construção de modelos digitais, } \\
\text { CAD, CAM e CAE. Tem a intenção de prever erros de projeto, auxiliar no } \\
\text { processo de orçamento, planejamento e produção do produto, objetivando a } \\
\text { redução de custo com protótipos físicos e tempo de desenvolvimento. }\end{array}$ & $\begin{array}{ll}{[1]} & \mathrm{e} \\
{[14] .} & \end{array}$ \\
\hline $\begin{array}{l}\text { Voz do } \\
\text { Consumidor }\end{array}$ & $\begin{array}{l}\text { Busca identificar e registrar as necessidades dos clientes, com o auxílio de } \\
\text { métodos de observação e coleta de dados, eliminação de necessidades similares } \\
\text { e filtragem, conversão de necessidades para requisitos de cliente. }\end{array}$ & $\begin{array}{l}{[1] \quad \mathrm{e}} \\
{[15] .}\end{array}$ \\
\hline Sala de Projetos & $\begin{array}{l}\text { Ambiente de trabalho onde a equipe de desenvolvimento de produto se reúne } \\
\text { com o propósito de atualizar o andamento dos processos realizados, tarefas } \\
\text { pendentes, possíveis soluções e demais informações pertinentes ao projeto. }\end{array}$ & $\begin{array}{l}{[16] \mathrm{e}} \\
{[17] .}\end{array}$ \\
\hline Fonte* & \multicolumn{2}{|l|}{$\begin{array}{l}\text { [1]: Dal Forno e Forcellini (2012); [2]: Zhu et al. (2016); [3]: Dekkers, Chang e } \\
\text { Kreutzfeldt (2013); [4]: Kincade, Regan e Gibson (2007); [5]: Schäfer e Sorensen (2010); } \\
\text { [6]: Becker e Wits (2015); [7]: Pinheiro e Toledo (2011); [8]: Patel, Chauhan e Trivedi } \\
\text { (2015); [9]: Tyagi et al. (2014); [10]: Heusner et al. (2015); [11]: Tyagi et al. (2015); [12]: } \\
\text { Silva Filho e Calado (2013); [13]: PMI (2008); [14]: Lindskog et al. (2015); [15]: } \\
\text { Rozenfeld et al. (2006); [16]: Terenghi et al. (2014) e [17]: Tezel et al. (2006). }\end{array}$} \\
\hline
\end{tabular}

Fonte: Adaptado de Prass (2017).

\section{PROPOSIÇÃO DO MODELO DE AVALIAÇÃO}

A construção do modelo de avaliação começa com a seleção das ferramentas do LPD (revisão da literatura), seguindo para a definição dos critérios de avaliação, ponderação dos critérios de avaliação, definição dos níveis de classificação e, por fim, a criação do instrumento de avaliação. As ferramentas do LPD selecionadas na literatura estão expostas na Tabela 1, presente no Capítulo 2 deste artigo. 
A visão geral da proposição do modelo de avaliação do emprego de ferramentas do Lean Product Development no PDP está apresentada na Figura 3.

Figura 3. Visão Geral da Proposição do Modelo de Avaliação. Fonte: Adaptado de Prass (2017).

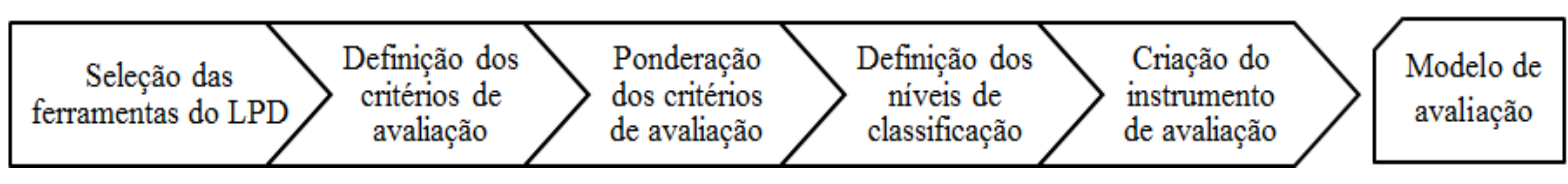

\subsection{Definição dos Critérios de Avaliação}

A definição dos critérios de avaliação ocorreu por meio da revisão da literatura, de reuniões e de discussões entre os pesquisadores, com o intuito de responder a seguinte pergunta norteadora: "quais são as condições para que uma empresa tenha um nível $\mathbf{X}$ (excelente, muito bom, satisfatório, ruim ou não aplica); no que se refere ao emprego de uma ferramenta Y (engenharia simultânea, mapeamento do fluxo de valor, [...])?” (PRASS, 2017).

Foram adotados 4 critérios de avaliação para cada ferramenta do LPD. Os 40 critérios de avaliação são apresentados nas Tabelas 2 e 3 .

Tabela 2. Critérios de Avaliação.

\begin{tabular}{|c|c|}
\hline Ferramenta & Critérios de Avaliação \\
\hline $\begin{array}{l}\text { Engenharia } \\
\text { Simultânea }\end{array}$ & $\begin{array}{l}\text { C1) A empresa aplica conceitos de engenharia simultânea. C2) Tem um processo de } \\
\text { desenvolvimento estruturado. C3) Utiliza ferramentas (softwares) para gerenciar o PDP. } \\
\text { C4) Busca melhorar o PDP continuamente. }\end{array}$ \\
\hline $\begin{array}{l}\text { Engenharia } \\
\text { Simultânea Baseada } \\
\text { em Conjuntos }\end{array}$ & $\begin{array}{l}\text { C1) A empresa aplica conceitos da engenharia simultânea baseada em conjuntos. C2) } \\
\text { Tem um processo estruturado, capaz de trabalhar com um conjunto de alternativas. C3) } \\
\text { Utiliza ferramentas (softwares) para gerenciar o PDP. C4) Busca melhorar o PDP } \\
\text { continuamente com conceitos de engenharia simultânea baseada em conjuntos. }\end{array}$ \\
\hline $\begin{array}{l}\text { Mapeamento do } \\
\text { Fluxo de Valor }\end{array}$ & $\begin{array}{l}\text { C1) A empresa utiliza o mapeamento do fluxo de valor para criar um mapa do estado } \\
\text { atual de um processo. C2) Cria um mapa de estado futuro. C3) Aplica a ferramenta para } \\
\text { melhorar os seus processos. C4) Tem o registro do tempo e do tempo efetivo de cada } \\
\text { atividade do processo, permitindo o cálculo da sua eficiência. }\end{array}$ \\
\hline $\begin{array}{l}\text { Gerenciamento } \\
\text { Visual }\end{array}$ & $\begin{array}{l}\text { C1) A empresa facilita o acesso à informação entre os envolvidos no PDP. C2) As } \\
\text { equipes de projeto buscam acessar as informações de desenvolvimento. C3) As equipes } \\
\text { de projeto buscam atualizar as informações de desenvolvimento. C4) Tem uma forma } \\
\text { padronizada de divulgar informações de desenvolvimento. }\end{array}$ \\
\hline Reporte A3 & $\begin{array}{l}\text { C1) A empresa utiliza o reporte A3 para apresentar propostas, auxiliar a tomada de } \\
\text { decisões e resolver problemas. C2) Registra-o em uma biblioteca de projetos. C3) Aplica } \\
\text { as soluções encontradas. C4) Acompanha a evolução das situações registradas. }\end{array}$ \\
\hline Biblioteca de Projeto & $\begin{array}{l}\text { C1) A empresa utiliza uma plataforma para organizar os arquivos registrados. C2) Os } \\
\text { arquivos são protegidos por nível hierárquico. C3) Realiza a cópia de segurança, } \\
\text { preservando o conhecimento. C4) Registra as lições aprendidas na biblioteca de projeto. }\end{array}$ \\
\hline Padronização & $\begin{array}{l}\text { C1) A empresa utiliza processos de desenvolvimento padronizados. C2) Busca por } \\
\text { projetos similares já realizados ao realizar o projeto informacional, conceitual e } \\
\text { preliminar. C3) Busca soluções padronizadas para os componentes do produto. C4) } \\
\text { Melhora os processos padronizados, com base nos conhecimentos obtidos. }\end{array}$ \\
\hline Simulação Virtual & $\begin{array}{l}\text { C1) A empresa utiliza ferramentas computacionais (CAD/CAM/CAE) durante o PDP. } \\
\text { C2) Capacita os funcionários nas ferramentas disponibilizadas regularmente. C3) As } \\
\text { ferramentas computacionais utilizadas têm compatibilidade de formato. C4) Busca a } \\
\text { reutilização de projetos, permitindo gerar geometrias padronizadas a partir de cotas. }\end{array}$ \\
\hline
\end{tabular}

Fonte: Adaptado de Prass (2017). 
Tabela 3. Critérios de Avaliação (continuação).

\begin{tabular}{|l|l|}
\hline Ferramenta & Critérios de Avaliação \\
\hline $\begin{array}{l}\text { Voz do } \\
\text { Consumidor }\end{array}$ & $\begin{array}{l}\text { C1) A empresa se preocupa em traduzir as necessidades do cliente nos requisitos de projeto. } \\
\text { C2) Utiliza ferramentas padronizadas para levantar todos os requisitos de projeto. C3) } \\
\text { Registra todas as informações geradas. C4) Capacita os profissionais em ferramentas que } \\
\text { auxiliam no levantamento dos requisitos de projeto. }\end{array}$ \\
\hline Sala de Projetos & $\begin{array}{l}\text { C1) A empresa utiliza conceitos da sala de projetos. C2) Expõe ferramentas gerenciais de } \\
\text { forma gráfica. C3) Tem um espaço físico próprio para reuniões de projeto. C4) Tem equipes } \\
\text { responsáveis pela atualização das informações de projeto. }\end{array}$ \\
\hline
\end{tabular}

Fonte: Adaptado de Prass (2017).

\subsection{Ponderação dos Critérios de Avaliação}

Para a ponderação dos critérios de avaliação empregou-se o método de tomada de decisão MACBETH (Measuring Attractiveness by a Categorical Based Evaluation Technique), que é uma abordagem que requer somente julgamentos qualitativos sobre os critérios. O método aplica um procedimento iterativo e inicial que compara dois critérios de cada vez, solicitando apenas um julgamento qualitativo. A consistência dos julgamentos é verificada e uma escala numérica é gerada a partir dos julgamentos do avaliador. De maneira similar, os pesos são definidos para os critérios de avaliação (BANA e COSTA et al, 2013).

Este procedimento iterativo foi realizado para cada uma das 10 ferramentas do LPD, onde os 4 critérios de avaliação de cada ferramenta são julgados par-a-par. Ao final, o peso dos 40 critérios são obtidos. A soma do peso dos 4 critérios é sempre igual a dez pontos $(10,00)$.

\subsection{Definição dos Níveis de Classificação}

A pontuação obtida é igual a soma do peso dos critérios atendidos. Ao atender os 4 critérios, a pontuação é máxima (10); e não atendendo nenhum dos 4 critérios, a pontuação é mínima (0). Definiu-se os níveis de avaliação: excelente, muito bom, satisfatório, ruim e não aplica, sendo que a maior pontuação em cada nível é definida como:

- Não aplica: zero;

- Ruim: peso do critério $\mathrm{C} 1$;

- Satisfatório: soma do peso dos critérios $\mathrm{C} 1$ e C2;

- Muito bom: soma do peso dos critérios C1, C2 e C3;

- Excelente: soma do peso dos critérios C1, C2, C3 e C4.

Desta maneira, as faixas de pontuação dos níveis de classificação podem ser representadas como exposto na Figura 4 (exemplificado para a engenharia simultânea).

Figura 4. Níveis de Classificação - Engenharia Simultânea. Fonte: Autoria Própria.

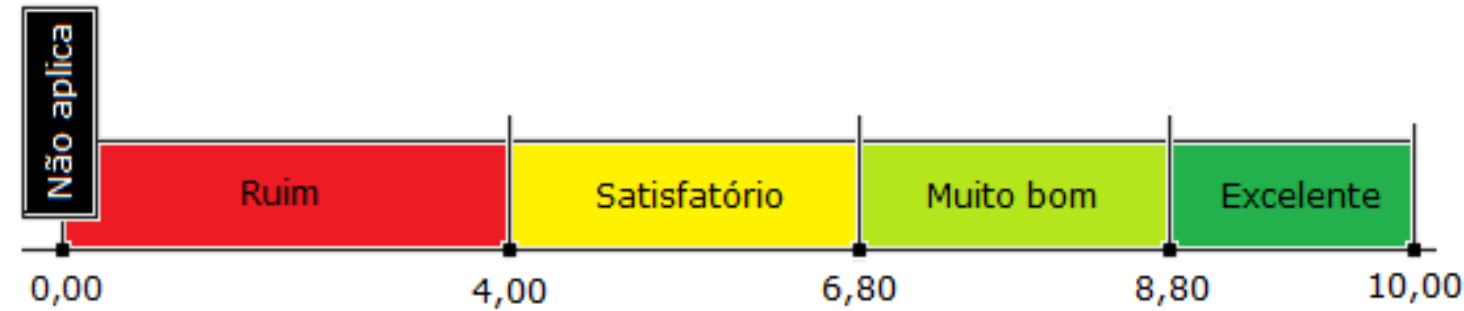

$\mathrm{Na}$ Figura 4 foram expostos os níveis de classificação para a engenharia simultânea, entretanto como os pesos dos critérios são diferentes para cada ferramenta, as pontuações dos níveis de classificação das ferramentas são diferentes, conforme apresentado na Tabela 4. 
Tabela 4. Pontuações dos Níveis de Classificação.

\begin{tabular}{|l|c|c|c|c|c|}
\hline Ferramentas do LPD & Não aplica & Ruim & Satisfatóno & Muito bom & Excelente \\
\hline Engenharia Simultânea & 0,00 & 4,00 & 6,80 & 8,80 & 10,00 \\
\hline Engenharia Simultânea Baseada em Conjuntos & 0,00 & 3,70 & 6,60 & 8,60 & 10,00 \\
\hline Mapeamento do Fluxo de Valor & 0,00 & 4,00 & 7,00 & 9,00 & 10,00 \\
\hline Gerenciamento Visual & 0,00 & 4,00 & 7,60 & 8,80 & 10,00 \\
\hline Reporte A3 & 0,00 & 3,30 & 5,90 & 8,20 & 10,00 \\
\hline Biblioteca de Projeto & 0,00 & 3,10 & 6,00 & 8,40 & 10,00 \\
\hline Padronização & 0,00 & 3,50 & 6,00 & 8,20 & 10,00 \\
\hline Simulação Virtual & 0,00 & 3,10 & 5,70 & 8,10 & 10,00 \\
\hline Voz do Consumidor & 0,00 & 3,10 & 5,90 & 8,10 & 10,00 \\
\hline Sala de Projetos & 0,00 & 3,20 & 5,90 & 8,10 & 10,00 \\
\hline Média & 0,00 & 3,50 & 6,34 & 8,43 & 10,00 \\
\hline
\end{tabular}

Fonte: Adaptado de Prass (2017).

\subsection{Criação do Formulário de Avaliação}

O último passo é a criação do formulário de avaliação. No caso, elaborou-se um formulário digital, que é um documento utilizado para formalizar a comunicação e coletar dados de maneira digital. Para a criação foi utilizada a ferramenta Google Forms. Para cada critério foi proposta uma pergunta de múltipla escolha, com três opções de respostas padronizadas: sim, às vezes, não ou não sei. "De acordo com a resposta inserida, uma porcentagem do peso de um critério de avaliação é atribuída na pontuação" (PRASS, 2017). De modo que, a resposta:

- Sim: atribuí $100 \%$ do peso do critério de avaliação;

- Às vezes: atribuí $50 \%$ do peso do critério de avaliação;

- Não ou não sei: atribuí $0 \%$ do peso do critério de avaliação.

Após atribuídas as pontuações em cada uma das ferramentas do LPD, as pontuações obtidas são classificadas e expostas em um gráfico do tipo radar com apoio de uma tabela.

\section{APLICAÇÃO DO MODELO DE AVALIAÇÃO}

Para aplicar o modelo de avaliação do emprego de ferramentas do LPD no PDP, primeiramente buscou-se empresas de diferentes setores que têm processos de desenvolvimento de produtos estruturados. Na sequência, dez (10) empresas atuantes no Sul do Brasil foram contatadas, das quais oito (8) participaram da avaliação, respondendo ao formulário digital. O perfil das empresas avaliadas é exposto na Tabela 5.

Tabela 5. Perfil das Empresas Avaliadas.

\begin{tabular}{|c|l|l|l|l|}
\hline Empresa & Principal Produto & Porte & País de origem & Abrangência \\
\hline 1 & Etiquetas e rótulos & Médio & Brasil & Nacional \\
\hline 2 & Moldes de injeção & Médio & Brasil & Nacional \\
\hline 3 & Escovas e pincéis & Grande & Brasil & Nacional \\
\hline 4 & Componentes automotivos & Grande & Brasil & Multinacional \\
\hline 5 & Produtos odontológicos & Grande & Brasil & Multinacional \\
\hline 6 & Projetos para indústria automotiva & Grande & Alemanha & Multinacional \\
\hline 7 & Equipamentos e produtos odontológicos & Grande & Alemanha & Multinacional \\
\hline 8 & Componentes automotivos & Grande & Alemanha & Multinacional \\
\hline
\end{tabular}

Fonte: Autoria Própria.

A seguir, os dados coletados foram processados, verificados quais critérios de avaliação foram atendidos em cada ferramenta do LPD, calculadas as pontuações e indicados os níveis 
de classificação. Por fim, relatórios de avaliação foram individualmente entregues às empresas, para demonstrar os resultados obtidos na avaliação.

\subsection{Resultados Obtidos}

A Figura 5 apresenta a pontuação média dos resultados obtidos pelas empresas, em cada ferramenta do LPD. No gráfico tipo radar, verifica-se os níveis de avaliação representados por cores e a pontuação média é demonstrada pela linha pontilhada. Na tabela da Figura 5, nota-se que a pontuação média do emprego das ferramentas (obtidas pelas oito empresas avaliadas) é de $\mathbf{6 , 4 9}$ pontos, classificado como muito bom.

Figura 5. Pontuação Média dos Resultados Obtidos. Fonte: Autoria Própria.

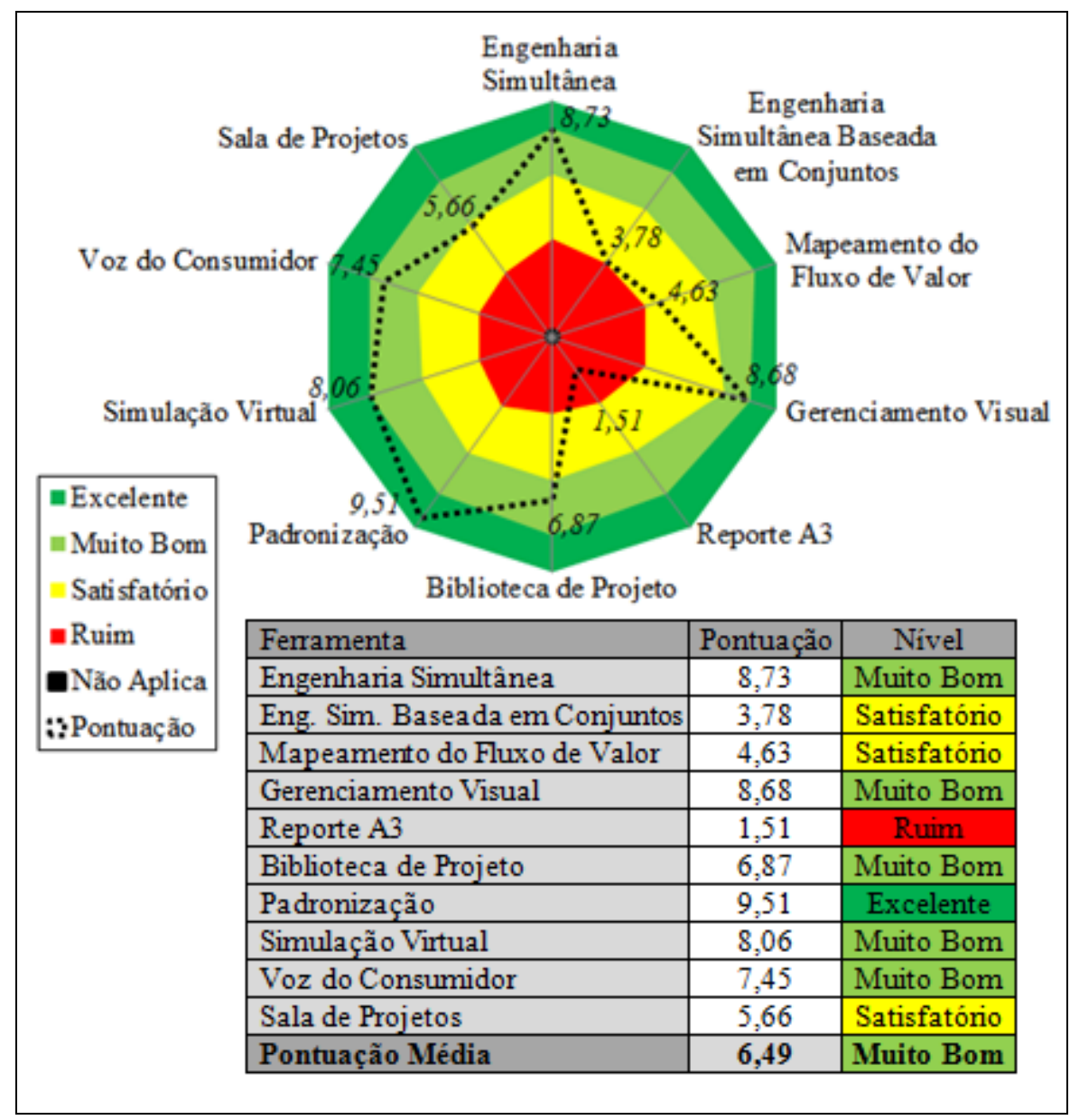

A ferramenta com a maior pontuação foi a padronização, que apresentou $\mathbf{9 5 \%}$ dos critérios de avaliação atendidos na média. E a ferramenta com menor pontuação foi o reporte $\mathbf{A 3}$, que teve apenas $\mathbf{1 5 \%}$ dos critérios de avaliação atendidos na média, isto porque apenas duas das empresas avaliadas empregam o reporte A3 no PDP.

\section{CONCLUSÕES}

Com os resultados obtidos com auxílio do modelo de avaliação proposto, pode-se concluir que existem ferramentas do LPD bem difundidas em empresas de diferentes setores, como a engenharia simultânea, o gerenciamento visual, a biblioteca de projetos, a padronização, a simulação virtual e a voz do consumidor. E por outro lado, têm-se ferramentas menos praticadas, como a engenharia simultânea baseada em conjuntos, o mapeamento do fluxo de valor, o reporte A3 e a sala de projetos. Isto demonstra que as ferramentas do Lean Product 
Development têm sido empregadas no PDP, porém estas ainda podem ser mais exploradas pela indústria em busca de aumentar a sua competitividade.

Deve-se notar que os dados obtidos com o auxílio do formulário de avaliação são provenientes da visão de um dos envolvidos no processo de desenvolvimento de produtos e pode não representar com precisão a realidade do PDP das empresas avaliadas.

Por fim, o modelo de avaliação proposto pode ser modificado e adaptado para atender diferentes modelos de referência para o desenvolvimento de produtos específicos, como moldes de injeção, compressores, refrigeradores, aeronaves e embarcações. Acrescentando ou removendo ferramentas do LDP, modificando os critérios de avaliação e ajustando os pesos dos critérios, de acordo com a necessidade apresentada. O que permite novas pesquisas sobre o emprego do Lean Product Development em diferentes setores da indústria.

\section{REFERÊNCIAS}

BANA e COSTA, C. A.; ANGULO-MEZA, L.; OLIVEIRA, M. D. O método Macbeth e aplicação no Brasil. ENGEVISTA, v15, n. 1, p. 3-27, 2013.

BECKER, J.M.J.; WITS, W.W. Enabling Lean Design Through Computer Aided Synthesis: The Injection Moulding Cooling Case. Procedia Cirp 37. p. 260-264, 2015.

DAL FORNO, A.J.; FORCELLINI, F.A. Lean product development: principles and practices. Product: Management \& Development, v10, n. 2, 2012.

DEKKERS, R.; CHANG, C.M.; KREUTZFELDT, J.. The interface between " product design and engineering", and manufacturing: a review of the literature and empirical evidence. Int. J. Production Economics, p. 316-333, 2013.

HEUSNER, R. et al. Aplicação da ferramenta mapeamento do fluxo de valor para identificação dos desperdícios do processo produtivo em uma empresa de reciclagem de plástico. Revista de Administração do Sul do Pará, Pará, v2, n. 3, p. 48-60, 2015.

KINCADE, D.H.; REGAN, C.; GIBSON, F.Y. Concurrent engineering for product development in mass customization for the apparel industry. International Journal Of Operations \& Production Management, p. 627-649, 2007.

LEÓN, H.C.M.; CROSS, J.A.. Lean Product Development Research: Current State and Future Directions. Current State And Future Directions: Engineering Management Journal, p. 29-51, 2011.

LINDSKOG, E. et al. Improving lean design of production systems by visualization support. Procedia CIRP 41, p. 602-607, 2016.

MACHADO, M.C. Gerenciamento estratégico do projeto e pensamento enxuto aplicados à metodologia de desenvolvimento de produtos. 2006. 265 f. Tese (Doutorado em Engenharia de Produção)- Universidade de São Paulo, São Paulo, Brasil, 2006.

MILAN, G.S.; REIS, Z.C.; COSTA, C.A. A implementação dos conceitos lean no processo de desenvolvimento de novos produtos. Qualitas Revista Eletrônica, v17, n. 1, 2015.

NAKAMURA, G. Inclusão dos conceitos enxutos nas fases iniciais do processo de projeto de produtos. 155 f. Dissertação (Mestrado em Engenharia Mecânica) - Universidade Federal de Santa Catarina, Florianópolis, Brasil, 2010. 
OEHMEN, J.; REBENTISCH, E. Waste in Lean Product Development. Lean Advancement Initiative Paper Series: Lean Product Development for Practitioners. Massachusetts Institute of Technology, 2010.

PATEL, N.; CHAUHAN, N.; TRIVEDI, P. Benefits of Value Stream Mapping as a Lean Tool Implementation Manufacturing Industries: A Review. International Journal for Innovative Research in Science \& Technology, p. 53-57, 2015.

PINHEIRO, L.M.P.; TOLEDO, J.C. Caracterização do sistema Lean (Toyota) de desenvolvimento de novos produtos para utilização em empresas brasileiras. $8^{\circ}$ Congresso Brasileiro de Gestão de Desenvolvimento de Produto, 2011.

PMI. Um guia do conhecimento em gerenciamento de projetos: Guia PMBOK®. 4. ed. Pensilvânia: Project Management Institute, Inc, 2008. 337 p.

PRASS, G. S. Modelo de avaliação do uso de práticas de desenvolvimento enxuto de produtos no processo de desenvolvimento de moldes de injeção. 2017. 167 f. Dissertação (Mestrado em Engenharia e Ciências Mecânicas) - Universidade Federal de Santa Catarina, Joinville, Brasil, 2017.

RAUCH, E.; DALlASEGA, P.; MATT, D.T. The way from Lean Product Development (LPD) to Smart Product Development (SPD). Procedia CIRP 50, p. 26-31, 2016.

ROZENFELD, H. et al. Gestão de Desenvolvimento de Produtos: uma referência para a melhoria do processo. São Paulo: Editora Saraiva, 2006. 542 p.

SALGADO, E.G. et al. Análise da aplicação do mapeamento do fluxo de valor na identificação de desperdícios do processo de desenvolvimento de produtos. Gestão da Produção, São Carlos, v16, n. 3, 2009.

SCHÄFER, H.; SORENSEN, D.J. Creating options while designing prototypes: value management in the automobile industry. Journal Of Manufacturing Technology Management, p. 721-742, 2010.

SILVA FILHO, O. S.; CALADO, R. Learning Supply Chain Management by PBL with A3 Report Support. 6th IFAC: Conference on Management and Control of Production and Logistics, p. 471-477, 2013.

TERENGHI, F. et al. Virtual Obeya: A new collaborative web application for running lean management workshops. 2014 International Conference on Engineering, Technology and Innovation, 2014.

TEZEL, A. et al. Visual management condition in highways construction projects in England. Proc. 24th Ann. Conf. of the Int'1. Group for Lean Construction, p. 133-142, 2016.

TYAGI, S. et al. Value stream mapping to reduce the lead-time of a product development process. Int. J. Production Economics, p. 202-212, 2014.

TYAGI, S. et al. Lean tools and methods to support efficient knowledge creation. International Journal of Information Management, p. 204-2014, 2015.

ZHU, A.Y. et al. The impact of organizational culture on Concurrent Engineering, Designfor-Safety, and product safety performance. Int. J. Production Economics, p. 69-81, 2016. 